Original Article

\title{
The Necessity of Anti-Tuberculosis Therapy after Resection of Pulmonary Tuberculous Nodules: A Single Center Retrospective Study
}

\author{
Chong Wang, MD, ${ }^{1}$ Yang Liu, MD, ${ }^{1}$ Haifeng Lin, ${ }^{2}$ Lei Yang, MD, ${ }^{1}$ Dongjie Yan, MD,${ }^{1}$ \\ Changfan Gong, MD, ${ }^{1}$ and Shuku Liu, $\mathrm{MD}^{1}$
}

\begin{abstract}
Purpose: To discuss the necessity of anti-tuberculosis therapy after resection of asymptomatic pulmonary tuberculous nodules: is postoperative anti-tuberculosis therapy is over-treatment?

Methods: This is a single-center retrospective study. Patients with solitary pulmonary nodule (SPN) and diagnosed as tuberculosis by pathology were included. Clinical features are collected. The primary end point is tuberculosis relapse and the secondary is adverse drug reactions. Patients are divided into two groups according to the acceptance of anti-tuberculosis treatment after operation (A: treated; B: untreated). Recurrence is diagnosed by multi-disciplinary discussion. The difference of recurrence rate will be compared and the incidence of adverse drug reactions in Group $A$ will be calculated.

Results: A total of 98 patients were enrolled, 66 in Group A and 32 in Group B. No significant difference between two groups was found in the past history of tuberculosis, erythrocyte sedimentation rate (ESR), T-spot positive rate, and the uptake value of 18F-glucose. No relapse of tuberculosis was found in both groups. The incidence of adverse drug reactions in Group A was $61 \%$ (40/66), and the rate of severe adverse reaction was $14 \%(9 / 66)$.

Conclusions: Postoperative recurrence of tuberculosis is rare, anti-tuberculosis treatment seems unnecessary for asymptomatic pulmonary tuberculous nodules. Adverse drug reactions should not be ignored.
\end{abstract}

Keywords: tuberculosis, nodules, pulmonary resection, recurrence, anti-tuberculosis treatment

${ }^{1}$ Minimally Invasive Treatment Center, Beijing Chest Hospital, Beijing, China

${ }^{2}$ Department of pathology, Beijing Chest Hospital, Beijing, China

Received: July 18, 2019; Accepted: October 29, 2019

Corresponding author: Shuku Liu, MD. Minimally Invasive Treatment Center, Beijing Chest Hospital, No.9, Beiguan Avenue, 101149, Beijing, China

Email: liushuku@sina.com

This work is licensed under a Creative Commons Attribution-NonCommercialNoDerivatives International License.

(C)2020 The Editorial Committee of Annals of Thoracic and Cardiovascular Surgery

\section{Introduction}

Surgical excision is important for the diagnosis and treatment of solitary pulmonary nodule $(\mathrm{SPN}) .{ }^{1)}$ Some patients with asymptomatic SPN were received pulmonary resection and the final pathology reported "caseous necrotic granuloma" or "massive necrosis and punctate calcification," etc. According to the microscopic morphology, they were diagnosed as pulmonary tuberculosis. The necessity of anti-tuberculosis therapy after resection of these pulmonary tuberculous granuloma is currently in dispute. The eighth edition of the textbook Surgery points 
Table 1 Basic information of patients

\begin{tabular}{lcccc}
\hline & N & Mean & Max. & Min. \\
\hline Gender (male/female) & $54 / 44$ & & & \\
Age & & $50 \pm 13.2$ & 76 & 20 \\
Follow-up time (months) & & $27.6^{\mathrm{a}}$ & 69.6 & 9.6 \\
History of previous TB (no/yes) & $89 / 9$ & & & \\
Surgical treatment & & & \\
$\quad$ Open/VATS & $35 / 63$ & & \\
$\quad$ Wedge/segmentectomy/lobectomy & $65 / 2 / 31$ & & \\
Postoperative anti-TB treatment (no/yes) & $32 / 66$ & & \\
Recurrence of TB & 0 & & \\
\hline
\end{tabular}

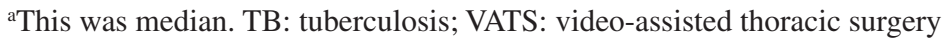

out that at least 6 months of anti-tuberculosis treatment is needed. ${ }^{2)}$ However, more and more asymptomatic tuberculous nodules have been found to make surgeons believe that there may be excessive treatment for adjuvant anti-tuberculosis after surgery. Because one of the criteria for tuberculosis cure is that the focus is completely absorbed or no Mycobacterium tuberculosis survive in the lesion. ${ }^{3)}$

We conduct this study to discuss the necessity of anti-tuberculosis therapy after resection of pulmonary tuberculous granuloma, by following up the postoperative anti-tuberculosis treatment and recurrence.

\section{Materials and Methods}

This is a single-center retrospective study. Patients met the following criteria were included: (1) asymptomatic SPN discovered by chest computed tomography (CT); (2) anti-fast staining of sputum smear was negative; (3) patients underwent surgical treatment; (4) no anti-tuberculosis treatment were conducted before operation; (5) tuberculosis or tuberculoma was diagnosed by pathology (molecular pathological methods included). Patients lost for follow-up and without complete imaging data were excluded from this study.

Past history, chest CT, positron emission tomography CT (PET-CT), tuberculosis-related examinations (tuberculosis infected T cells spot test [T-SPOT] and erythrocyte sedimentation rate [ESR]), operation and postoperative anti-tuberculosis drugs were collected from clinical database.

The follow-up focused on the following: (1) the species and period of anti-tuberculosis treatment; (2) drug-related adverse reactions; (3) mental disorders from administration of anti-tuberculosis drugs; and (4) postoperative chest CT examination. The main end point was tuberculosis relapse and the secondary one was adverse drug reaction. Patients were divided into two groups: group A (patients received anti-tuberculosis treatment after operation) and group B (patients without anti-tuberculosis treatment). The difference of tuberculosis recurrence between two groups was analyzed. The incidence of adverse drug reactions was calculated in group A.

Tuberculosis recurrence were defined as follows: (1) new nodules around the surgical site with a typical tuberculosis imaging manifestations; (2) patients had fever, cough, and other tuberculosis-related symptoms and new lesions could be found in imaging examination, and anti-tuberculosis treatment was effective; and (3) antifast staining of sputum smear was positive.

Measurement data between two groups were compared by t-test or rank sum test. Categorical data were analyzed by $\chi^{2}$ test or fisher exact test. The difference was significant when $\mathrm{p}<0.05$.

\section{Results}

From April 2011 to December 2018, a total of 98 patients were eligible for analysis in this study. Among them, there were 54 men and 44 women, with an average age of 50. The median follow-up time was 27.6 months. There were 66 patients in group A and 32 patients in group B. There was no recurrence of tuberculosis either in group A and B (Table 1). So, there was no need for propensity score matching or factor analysis.

We compared the previous history of tuberculosis, ESR, TB-DNA and T-SPOT positive rate, standard uptake value (SUV) level, body mass index (BMI) between two groups. The positive rate of TB-DNA and acid-fast stain in group A was significantly higher than group B, and the diameter of lesion in group A was significantly larger than that of group B (Table 2). 
Table 2 Comparison of clinical features between groups A and B

\begin{tabular}{lcccc}
\hline & $\begin{array}{c}\text { Group A } \\
\mathbf{n}=\mathbf{6 6}\end{array}$ & $\begin{array}{c}\text { Group B } \\
\mathbf{n =}=\mathbf{3 2}\end{array}$ & $\chi^{\mathbf{2}}$ & $\mathbf{p}$ \\
\hline Past history of TB & & & & $0.264^{\mathrm{a}}$ \\
$\quad$ Yes & 8 & 1 & & \\
$\quad$ No & 58 & 31 & & \\
ESR & 9 & 3 & & $0.739^{\mathrm{a}}$ \\
$\quad$ Positive & 38 & 19 & & \\
$\quad$ Negative & 19 & 10 & & \\
NR & & & 0.025 & 0.874 \\
T-SPOT & 31 & 15 & & \\
$\quad$ Positive & 23 & 12 & & \\
$\quad$ Negative & 22 & 5 & & \\
$\quad$ NR & 48 & 11 & 13.231 & $<0.001$ \\
TB-DNA/acid-fast stain & 18 & 21 & & \\
$\quad$ Positive & & & & \\
$\quad$ Negative & 30 & 20 & 0.181 & 0.670 \\
PET-CT & 15 & 8 & & \\
$\quad$ Low uptake & 21 & 4 & & $0.007^{\mathrm{b}}$ \\
$\quad$ High uptake & $2.3 \pm 1.1$ & $1.7 \pm 0.8$ & & $0.477^{\mathrm{b}}$ \\
$\quad$ NR & $23.4 \pm 2.8$ & $24.1 \pm 3.5$ & & \\
Size in CT(cm) & & & & \\
BMI &
\end{tabular}

${ }^{a}$ Based on Fisher exact test. ${ }^{b}$ t-test. BMI: body mass index; ESR: erythrocyte sedimentation rate; NR: not report; TB: tuberculosis; T-SPOT: tuberculosis infected T cells spot test; PET-CT: positron emission tomography CT

We used 10 tags to describe imaging features of each lesion in $\mathrm{CT}$, such as punctuated calcification, spiculation, irregular shape, pleural traction, lobulation, vacuoles, satellite foci, calcified lymph nodes, ground-glass opacity, and round-like shape. One lesion could have multiple tags. We compared the frequency of different tags in two groups and the result showed that spiculation, oval shape, pleural retraction, and irregular shape were the most common tags (from more to less) both in two groups. That was to say the imaging features were similar between groups A and B (Table 3).

In group A, we found that (Table 4) (1) four-drug combination chemotherapy including isoniazid, rifampicin/rifapentine, ethambutol, and pyrazinamide was the most commonly used, but there were many other combination of drugs; (2) the course of anti-tuberculosis varied greatly from 1 month to more than 1 year. And 6 months was the most common course of treatment; (3) about $24 \%$ (16/66) patients stopped administration without doctor's orders because they did not know when to stop the drugs; (4) $61 \%$ (40/66) of patients had adverse drug reactions. The most common adverse reaction of antituberculosis drugs was elevation of liver enzymes and uric acid. Most of them could be improved after corresponding treatment. But 23\% (9/40) had severe adverse reactions, which lead to the direct withdrawal of their own drugs; (5) almost half (31/66) of the patients were disgust for taking anti-tuberculosis medicine and $26 \%$ $(8 / 31)$ of them were severe.

\section{Discussion}

The study is derived from specific clinical problems. Many thoracic surgeons believe that anti-tuberculosis treatment after resection of small tuberculoma is unnecessary because these small tuberculosis, like early-stage cancer, could be cured by surgical resection without postoperative adjuvant treatment. But many tuberculosis physicians believe that tuberculosis is a systemic disease and requires regular anti-tuberculosis treatment. So, we conduct this study to answer this question. The most effective research design is a prospective randomized controlled trials study. Given the potential ethical risks and no past studies for references, we conduct this retrospective study first to find some preliminary evidence for future prospective research.

In this study, the diagnosis of tuberculosis was mainly based on the pathological results. Patients can be divided into two categories. The first one's diagnosis was confirmed by detection of TB-DNA through fluorescent 
Table 3 Imaging features of lesions in two groups

\begin{tabular}{lcccccc}
\hline & Group A (n) & Group A $(\boldsymbol{\%})$ & Group B (n) & Group B (\%) & $\boldsymbol{\chi}^{\mathbf{2}}$ & P \\
\hline Spiculation & 36 & 55 & 18 & 56 & 0.025 & 0.874 \\
Oval & 33 & 50 & 19 & 59 & 0.761 & 0.383 \\
Pleural retraction & 29 & 44 & 13 & 41 & 0.097 & 0.756 \\
Irregular & 27 & 41 & 10 & 31 & 0.856 & 0.355 \\
Lobulation & 20 & 30 & 11 & 34 & 0.165 & 0.684 \\
Calcification & 22 & 33 & 9 & 28 & 0.27 & 0.603 \\
Satellite foci & 22 & 33 & 2 & 6 & 1.358 & 0.244 \\
Vacuole sign & 8 & 12 & 5 & 16 & $0.368^{\mathrm{a}}$ \\
Ground glass opacity & 4 & 6 & 0 & 0 & $0.124^{\mathrm{a}}$ \\
Calcified lymph node & 1 & 2 & & $0.484^{\mathrm{a}}$ \\
\hline
\end{tabular}

${ }^{a}$ From Fisher exact test

Table 4 Treatment of anti-tuberculosis after operation

\begin{tabular}{|c|c|c|}
\hline & & $\mathbf{N}$ \\
\hline \multicolumn{3}{|l|}{ Combination of anti-TB drugs } \\
\hline & INH, RFP/Rft, EMB, PZA & 38 \\
\hline & INH, RFP/Rft, EMB & 20 \\
\hline & Others & 8 \\
\hline \multicolumn{3}{|l|}{ Course (month) } \\
\hline & $<6$ & 12 \\
\hline & 6 & 22 \\
\hline & $7-11$ & 6 \\
\hline & 12 & 6 \\
\hline & $>12$ & 7 \\
\hline & Unknown & 13 \\
\hline \multicolumn{3}{|c|}{ Withdrawal of anti-TB treatment based on } \\
\hline & Doctor's advice & 28 \\
\hline & Patients themselves & 14 \\
\hline & Under treatment & 8 \\
\hline & Unknown & 16 \\
\hline \multicolumn{3}{|l|}{ Adverse reaction } \\
\hline & No & 26 \\
\hline & Elevated hepatic enzyme & $12\left(4^{\mathrm{a}}\right)$ \\
\hline & Elevated uric acid & $11\left(3^{a}\right)$ \\
\hline & Gastrointestinal reaction & $7\left(2^{a}\right)$ \\
\hline & Allergy & 2 \\
\hline & Hypoleukopenia & 2 \\
\hline & Others & 6 \\
\hline \multicolumn{3}{|c|}{ Psychological disorder of anti-TB treatment } \\
\hline & No & 24 \\
\hline & Resisted to treatment & $31\left(8^{\mathrm{a}}\right)$ \\
\hline & Unknown & 11 \\
\hline
\end{tabular}

aNumber of severe patients. EMB: ethambutol; INH: isoniazid; RFP: rifampin; Rft: rifapentine; PZA: Pyrazinamide; TB: tuberculosis

staining polymerase chain reaction (PCR). The other one lacked molecular evidence of tuberculosis but it had typical tuberculous epithelioid granulomatous patterns (epithelioid cells, Langerhans, and caseous necrosis), which were caused by cell-mediated immunity and delayed hypersensitivity. ${ }^{4)}$ In China, tuberculosis is divided into three types: latent infection of Mycobacterium tuberculosis, active tuberculosis, and non-active tuberculosis. The latter one is defined as: no tuberculosis-related clinical symptoms, negative bacteriological examination, certain imaging features (solitary or multiple calcified lesions; cord-like lesions; induration lesions; purification cavity; pleural thickening, adhesion or calcification). However, Guidelines from The World Health Organization (WHO) and 
National Institute for Health and Care Excellence (NICE) divide tuberculosis into two types: active and latent. Latent tuberculosis is defined as state of persistent immune response to stimulation by Mycobacterium tuberculosis antigens without evidence of clinically manifested active tuberculosis. ${ }^{5,6)}$ According to the above definitions, the research objectives in this study are more consistent with non-active tuberculosis or latent tuberculosis.

In the WHO guideline, anti-tuberculosis treatment should be performed if the patient has a risk of progressing to active tuberculosis. The main risk factors are as follows: close contact history of tuberculosis, human immunodeficiency virus (HIV) infection, less than 5-yearold, alcoholism, drug abuse, organ transplantation history, hematological malignancies, chemotherapy history, diabetes, chronic kidney disease, tumor necrosis factor (TNF) treatment history, and cirrhosis. Of course, most of the patients in this study do not have these risk factors. That is to say that anti-tuberculosis seems unnecessary.

Some studies on the surgical treatment of tuberculosis reported "conventional" anti-tuberculous therapy after resection. However, these studies focus on whether the operative method was reliable, rather than the long-term prognosis of surgery. ${ }^{7-9)}$ Conventional antituberculosis treatment after surgery lacks evidencebased medicine's support because the experience came from the surgical treatment of previous active tuberculosis, such as drug-resistant tuberculosis and cavitary tuberculosis. ${ }^{10)}$ In 2014, an India study with an average follow-up of 9 years showed that 43 cases of solitary pulmonary tuberculosis had no recurrence after resection, but all patients received anti-tuberculosis treatment. ${ }^{11)}$ As a result of the inability to obtain the full text, the details of the study were unknown. At present, WHO defines the standard of clinical cure for tuberculosis as follows: sputum continuous negative, obvious absorption or improvement of lesions, cavity closure, or reduction for more than half a year. ${ }^{3)}$ For patients in this study, tuberculosis was in a state of dormancy. Surgical removal of this kind of latent tuberculosis seems to be a cure, so there is no need for anti-tuberculosis treatment in theory. Our follow-up results confirmed that patients who did not have anti-tuberculosis treatment had no recurrence of tuberculosis.

By comparing the clinical data of the two groups, we found that the positive rate of TB-DNA in the antituberculosis treatment group was significantly higher than the group without anti-tuberculosis, which may be the evidence for the treatment of tuberculosis patients in clinic.
Therefore, the adverse reaction of anti-tuberculosis drugs should be paid enough attention. In the WHO guideline for latent tuberculosis, it is mentioned that "The potential benefit of treatment needs to be carefully balanced against the risk of drug-related adverse events." ${ }^{5}$ During follow-up, more than half of the patients had various adverse reactions. Meanwhile, about $50 \%$ patients refused to take drugs and felt fear of medication during the period of anti-tuberculosis treatment. So, for these patients, anti-tuberculosis treatment had caused physical and mental harm. We also found that there was a great difference in anti-tuberculosis combination and course of treatment. Patient's compliance was not strong enough. A lot of patients stopped taking medicine by themselves, or did not know when to stop for lack of doctor's guidance. This illustrates that many doctors are not clear about this problem, and the treatment is more arbitrary, which cannot form a common understanding and standardized treatment.

We need to point out that this retrospective study has some limitations. First, follow-up time is not long enough because there may be a long interval between tuberculosis recurrence and resection. Second, the total number of cases is still small and the sample size of the two groups is quite different, which may be related to selective bias in tuberculosis hospitals. Therefore, multiple-center clinical trials are needed to verify the difference.

\section{Conclusion}

Postoperative recurrence of tuberculosis is very rare, and anti-tuberculosis treatment seems unnecessary for asymptomatic pulmonary tuberculous nodules. Adverse drug reactions should not be ignored. However, multiplecenter prospective studies are needed for further research.

\section{Disclosure Statement}

This study has no conflict of interest.

\section{References}

1) Ost D, Fein AM, Feinsilver SH. Clinical practice. The solitary pulmonary nodule. N Engl J Med 2003; 348: 2535-42.

2) Wang J. Surgical treatment of pulmonary infectious diseases. In: Chen XP, Wang JP eds. Surgery, 8th ed. Beijing: People's Health Publishing House, 2013; 278-9, [in Chinese]. 
3) World Health Organization. The Treatment of Tuberculosis: Guidelines. Geneva: World Health Organization; 2010. Available from: https://www.ncbi.nlm.nih. gov/books/NBK138748/.

4) Reza JM, Jean D. Tuberculosis and atypical mycobacterial diseases. In: Patterson GA, Croper JD, Destourices J, Iudcetch AD, Rke TW, eds. Pearson's Thoracic $\&$ Esophageal Surgery. 3rd ed. Amsterdam: Elsevier, 2008; 505.

5) World Health Organization. Guidelines on the Management of Latent Tuberculosis Infection. Geneva: World Health Organization; 2015. Available from: http://www.who.int/tb/publications/latent-tuberculosisinfection/en/.

6) National Institute for Health and Care Excellence. Tuberculosis. London: National Institute for Health and Care Excellence; 2016. Available from: http:// www.nice.org.uk/guidance/ng33.
7) Hsu KY, Lee HC, Ou CC, et al. Value of video-assisted thoracoscopic surgery in the diagnosis and treatment of pulmonary tuberculoma: 53 cases analysis and review of literature. J Zhejiang Univ Sci B 2009; 10: 375-9.

8) Yablonskii P, Kudriashov G, Vasilev I, et al. Robotassisted surgery in complex treatment of the pulmonary tuberculosis. J Vis Surg 2017; 3: 18.

9) Bagheri R, Haghi SZ, Rajabi MT, et al. Outcomes following surgery for complicated tuberculosis: analysis of 108 patients. Thorac Cardiovasc Surg 2013; 61: 154-8.

10) Man MA, Nicolau D. Surgical treatment to increase the success rate of multidrug-resistant tuberculosis. Eur J Cardiothorac Surg 2012; 42: e9-12.

11) Laisaar T, Viiklepp P, Hollo V. Long-term follow-up after thoracoscopic resection of solitary pulmonary tuberculoma. Indian J Tuberc 2014; 61: 51-6. 